\title{
Ground states of spin-1 bosons in asymmetric double wells
}

\author{
D. W. S. Carvalho, A. Foerster, and M. A. Gusmão \\ Instituto de Física, Universidade Federal do Rio Grande do Sul, Caixa Postal 15051, 91501-970 Porto Alegre, Brazil
}

(Received 22 December 2014; published 6 March 2015)

\begin{abstract}
In this work we investigate the different states of a system of spin-1 bosons in two potential wells connected by tunneling, with spin-dependent interaction. The model utilizes the well-known Bose-Hubbard Hamiltonian, adding a local interaction term that depends on the modulus of the total spin in a well, favoring a high- or low-spin state for different signs of the coupling constant. We employ the concept of fidelity to detect critical values of parameters for which the ground state undergoes significant changes. The nature of the states is investigated through evaluation of average occupation numbers in the wells and of spin correlations. A more detailed analysis is done for a two-particle system, but a discussion of the three-particle case and some results for larger numbers are also presented.
\end{abstract}

DOI: 10.1103/PhysRevA.91.033608

PACS number(s): 03.75.Mn, 67.85.Fg, 67.85.Hj

\section{INTRODUCTION}

The experimental realization of Bose-Einstein condensates (BEC) in dilute atomic gases [1-3] is one of the most exciting recent achievements in physics, and research associated with this peculiar state of matter has flourished in recent years. One remarkable development in this context is the realization of spinor Bose gases in optical lattices. In contrast to a magnetic trap, where spins are frozen, in an optical trap the atoms keep their spin degrees of freedom. Several experimental groups have successfully created spinor BECs of ${ }^{23} \mathrm{Na}[4,5]$ and ${ }^{87} \mathrm{Rb}$ atoms [6-8]. Spinor gases exhibit richer quantum effects than their single-component counterparts, and allow us to investigate mesoscopic magnetism.

These experimental developments have stimulated extensive study of related theoretical models [9-24]. In particular, the behavior of spin-1 bosons in a double-well potential can be described by a variant of the two-site Bose-Hubbard Hamiltonian [25] including spin-dependent interactions $[11,23]$ that affect physical properties of the system. Of particular interest is the case where the number of atoms is small, motivated by the recent successful experimental trapping of few atoms with high control and precision [26-28]. Remarkably, the experimental preparation of only two interacting particles in a double well has been reported [29], and in principle the extension to three particles is feasible with the state-of-the-art experiments [30]. These experimental achievements have generated an intense theoretical effort in few-body quantum systems (see, for instance, Refs. [31-41]). Despite their simplicity, they still constitute a very challenging research field.

In this work we investigate the ground-state properties of a few spin-1 bosons in a double well. Such systems can be viewed as building blocks of optical lattices with cold atoms that can be in three different hyperfine states. Besides the usual Hubbard-type repulsion, the model includes a spin-dependent attractive interaction between the particles, which may favor the establishment of a high- or low-spin state in each of the wells depending on the sign of the coupling constant of this interaction. Our basic goal is to study changes in the characteristics of the ground state induced by variations of the model parameters.

The paper is organized as follows. In Sec. II, we discuss the Hamiltonian and its diagonalization. A detailed analysis of the two-particle system is developed in Sec. III, where we also introduce the tools that we use to obtain information about relevant properties. Section IV shows relatively detailed results for the three-boson system and discusses general trends for larger numbers, using the cases of four and five particles as examples. Final remarks are presented in Sec. V.

\section{MODEL AND MATRIX REPRESENTATION}

Following Ref. [23], we write a variant of the Bose-Hubbard Hamiltonian for a system composed of two wells, at positions $L$ (left) and $R$ (right), as

$$
\begin{aligned}
H= & \epsilon\left(n_{L}-n_{R}\right)-t \sum_{\sigma}\left(a_{L \sigma}^{\dagger} a_{R \sigma}+a_{R \sigma}^{\dagger} a_{L \sigma}\right) \\
& +\frac{1}{2} U \sum_{i=L, R} n_{i}\left(n_{i}-1\right)+\frac{1}{2} U^{\prime} \sum_{i=L, R}\left(\mathbf{S}_{i}^{2}-2 n_{i}\right),
\end{aligned}
$$

where $a_{i \sigma}^{\dagger}$ and $a_{i \sigma}$ are the creation and annihilation operators of a boson in a given well $(i=L, R)$ and in the spin state $\sigma=$ $\{-1,0,1\} ; n_{i}$ and $\mathbf{S}_{i}$ are the number and total-spin operators for each well. We assume a single level per well in the zerotunneling limit $(t=0)$, with energies $\pm \epsilon$. This means that $\epsilon$ is an asymmetry (or tilt) parameter, since the wells are identical only for $\epsilon=0$. All interactions in the model are local. Apart from the usual (repulsive) Hubbard interaction $U$, the last term in Eq. (1) describes a spin-dependent interaction with coupling constant $U^{\prime}$. It should be noted that this term contributes only when more than one particle is present in the same well, in which case low- and high-spin states are favored for $U^{\prime}>$ 0 and $U^{\prime}<0$, respectively. The number and spin operators appearing in the Hamiltonian are given by

$$
n_{i}=\sum_{\sigma} a_{i \sigma}^{\dagger} a_{i \sigma}, \quad \mathbf{S}_{i}=\sum_{\sigma \sigma^{\prime}} a_{i \sigma}^{\dagger} \mathbf{T}_{\sigma \sigma^{\prime}} a_{i \sigma^{\prime}},
$$

where $\mathbf{T}=T_{x} \hat{\mathbf{x}}+T_{y} \hat{\mathbf{y}}+T_{z} \hat{\mathbf{z}}$, in terms of the usual spin-1 matrices.

From Eq. (1) we can see that the total number of particles $\left(N_{t}=N_{L}+N_{R}\right)$, the total spin $\left(\mathbf{S}_{t}=\mathbf{S}_{L}+\mathbf{S}_{R}\right)$, as well as any component of the latter (which we choose to be $S_{t}^{z}$ ) are conserved quantities. It is natural to investigate the properties of a system with a fixed number of particles. Moreover, since 
the energies do not depend on the value of $S_{t}^{z}$, we can restrict our analysis to the subspace with $S_{t}^{z}$ eigenvalue equal to zero, as this subspace is always present for any number of spin-1 particles.

A convenient basis is provided by a set of vectors of the form $\left|\left\{N_{L}, N_{R}\right\},\left\{S_{L}, S_{R}\right\}, S_{t}\right\rangle$, labeled by the number and spin values in each well, and the total spin $S_{t}$. Bosonic symmetry imposes that $S_{i}+N_{i}$ must be an even integer [23] $(i=L, R)$. This is an interesting basis because it explicitly separates subspaces of different values of $S_{t}$, which are not connected by the Hamiltonian. However, to deal with the tunneling term it is better to use appropriate symmetric combinations of vectors of type $\left|N_{L}^{(0)}, N_{L}^{(1)}, N_{L}^{(-1)} ; N_{R}^{(0)}, N_{R}^{(1)}, N_{R}^{(-1)}\right\rangle$, specifying the number of particles per spin state in each well. Since these two sets of vectors are related by Clebsh-Gordon coefficients, it is straightforward to generate any matrix elements.

All results that we discuss here were obtained starting from exact diagonalization of the Hamiltonian matrix for a given set of model parameters and particle number. The on-site Coulomb repulsion $U$ was kept fixed and chosen to be the energy unit. Hence, in what follows we set $U=1$, it being implicitly assumed that any quantity with dimension of energy is expressed in units of $U$.

\section{TWO-PARTICLE SYSTEM}

We begin by investigating the energy spectrum for the simplest case, $N_{t}=2$, with emphasis on changes in the ground state when varying the parameters of the Hamiltonian.

As an example, Fig. 1 shows the energy eigenvalues for two bosons as functions of the asymmetry parameter $\epsilon$, for moderately weak tunneling $(t=0.1)$ and spin-dependent coupling $\left(U^{\prime}=0.3\right)$. For comparison, the top panel shows the case of decoupled wells $(t=0)$. We recall that the local energy levels are $\epsilon$ and $-\epsilon$ for left $(L)$ and right $(R)$ wells, respectively, which can be related to their depths. Thus, $\epsilon<0$ means that the $L$ well is deeper. The inversion symmetry around $\epsilon=0$ is noticeable in the plots.

From Fig. 1 it is clear that there are values of $\epsilon$ near which the ground-state changes. These points occur at level crossings in the absence of tunneling, and the degeneracy lifting is stronger as tunneling increases.

\section{A. Ground-state changes monitored by fidelity}

Ground-state changes in finite-size systems may be viewed as precursors of quantum phase transitions (QPTs) in the macroscopic limit. Among the usual techniques to detect QPTs, the fidelity of two ground states corresponding to different sets of parameters can be used unambiguously for finite-size systems. This is a concept derived from quantuminformation theory and measures the similarity between two quantum states. Although there are generalized definitions [42], the simplest one, which serves our purposes, is

$$
\mathcal{F}(\psi, \phi)=|\langle\psi \mid \phi\rangle|
$$

It defines the fidelity between any two states of the Hilbert space as the absolute value of their scalar product. For normalized states, $0 \leqslant \mathcal{F} \leqslant 1$.
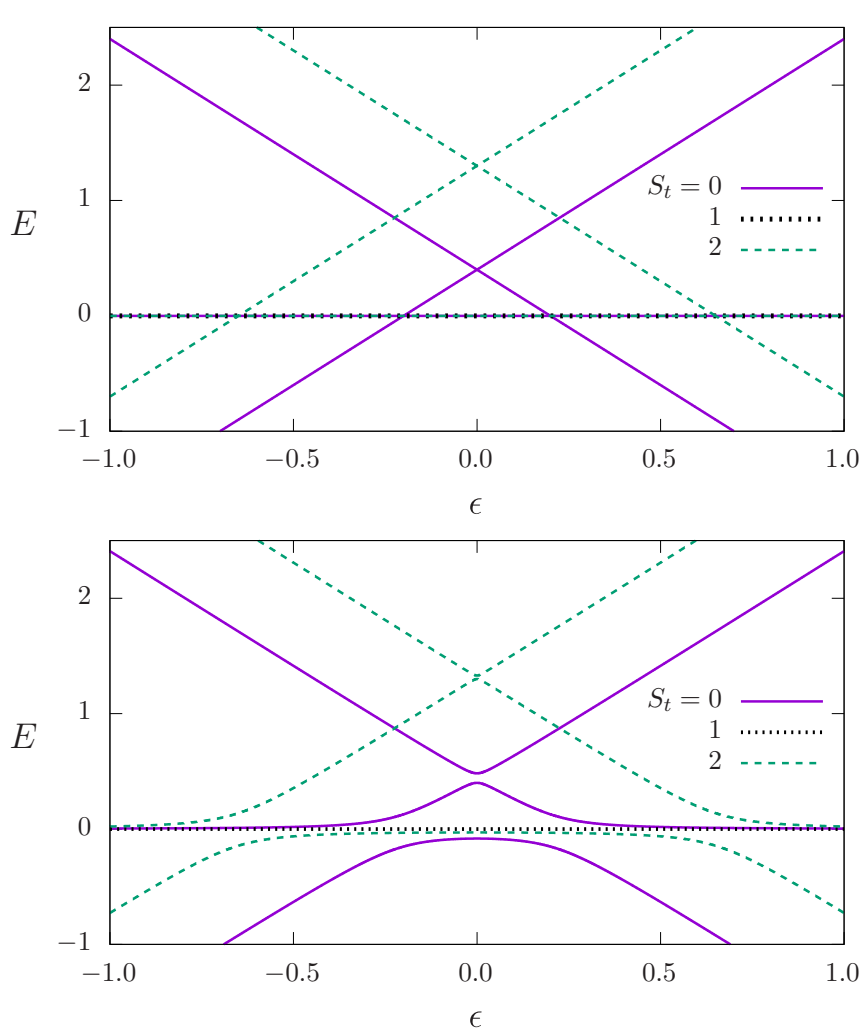

FIG. 1. (Color online) Energy eigenvalues as functions of $\epsilon$, for $N_{t}=2$ and $U^{\prime}=0.3$. The top panel shows decoupled wells $(t=0)$, while the bottom panel corresponds to moderately weak tunneling $(t=0.1)$.

In our case, judging from Fig. 1, an appropriate control parameter is $\epsilon$, so that we define the fidelity

$$
\mathcal{F}_{\epsilon}\left(\epsilon ; N_{t}, t, U^{\prime}\right)=\left|\left\langle\epsilon-\delta, N_{t}, t, U^{\prime} \mid \epsilon+\delta, N_{t}, t, U^{\prime}\right\rangle\right|,
$$

where $\delta$ is small in the scale of $\epsilon$ values, and the notation for the ground-state vector also includes all quantities that are kept fixed. Later on we are interested in the effect of varying the spin-dependent interaction $U^{\prime}$, in which case a more convenient choice of fidelity is

$$
\mathcal{F}_{U^{\prime}}\left(\epsilon, N_{t}, t ; U^{\prime}\right)=\left|\left\langle\epsilon, N_{t}, t, U^{\prime}-\delta \mid \epsilon, N_{t}, t, U^{\prime}+\delta\right\rangle\right| .
$$

Characteristic behavior of $\mathcal{F}_{\epsilon}$ for two particles is shown in Fig. 2. The smoother curve corresponds to the system parameters used in Fig. 1. Clearly defined minima are observed at values of the control parameter for which a substantial change of ground state occurs. These minima become much sharper when the tunneling is reduced, as shown by the dashed curve, for which they appear at different $\epsilon$ values because a different $U^{\prime}$ was chosen.

\section{B. Occupation number and spin correlations}

Although the fidelity finds parameter values for which the ground sate changes, it does not give direct information about the nature of states. For this we need to evaluate (average values of) relevant physical quantities. An obvious one is the occupation number of each well [23]. Also interesting is the spin correlation function, usually employed to study magnetic properties of solids, as they are directly 


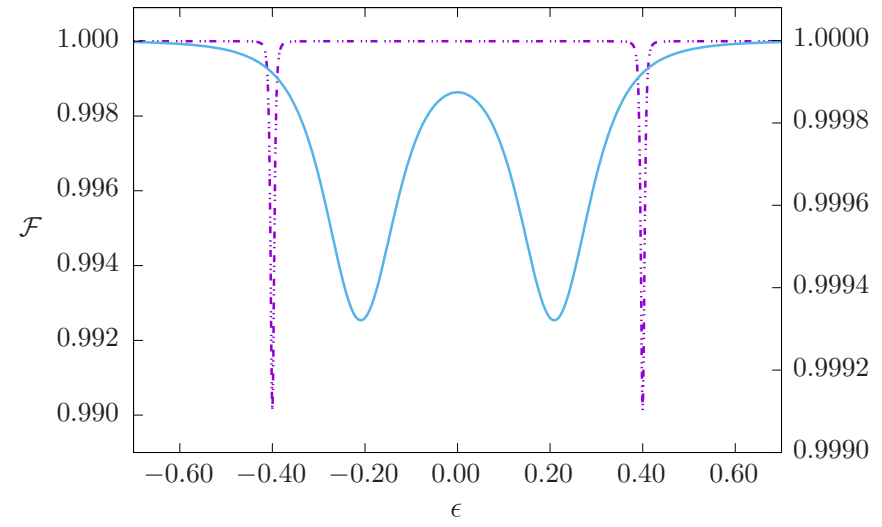

FIG. 2. (Color online) Ground-state fidelity as a function of the asymmetry parameter $\epsilon$ for $N_{t}=2$, with two sets of values of tunneling amplitude and spin-dependent coupling: $t=0.1, U^{\prime}=0.3$ (solid line, right y axis), and $t=0.005, U^{\prime}=0.1$ (dashed, left y axis).

associated with magnetic susceptibility. The latter not only gives the response to an applied field but serves to signal the establishment of magnetic order. The concept of magnetic order does not make sense in a small system as the one that we are studying. However, spin correlation functions can give important information about the nature of the ground state with respect to relative orientations of the spins.

We define the spin correlation function between the two wells as

$$
C\left(\epsilon, N_{t}, t, U^{\prime}\right)=\left\langle\epsilon, N_{t}, t, U^{\prime}\left|\mathbf{S}_{L} \cdot \mathbf{S}_{R}\right| \epsilon, N_{t}, t, U^{\prime}\right\rangle,
$$

where $\mathbf{S}_{L}$ and $\mathbf{S}_{R}$ denote the total spin operator associated to the left and right wells, respectively.

Figure 3 shows typical behavior of the right-well occupation number and interwell spin correlation function for two particles, in the same range of relative depths as in Fig. 2. The staircase behavior of $N_{R}$ is easy to understand on the basis of a competition between the on-site repulsion, that tends to keep particles apart, and the energy asymmetry, that favors occupation of the deepest well. The role of $U^{\prime}$ is to select spin

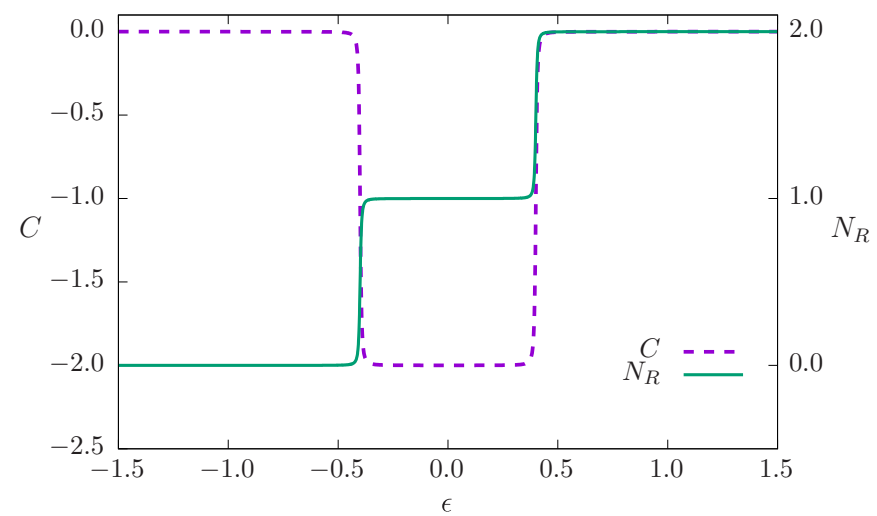

FIG. 3. (Color online) Occupation number of the $R$ well (solid line) and interwell spin correlation function (dashed) as a function of $\epsilon$ for two particles, with the same parameters that show sharp minima in Fig. 2.

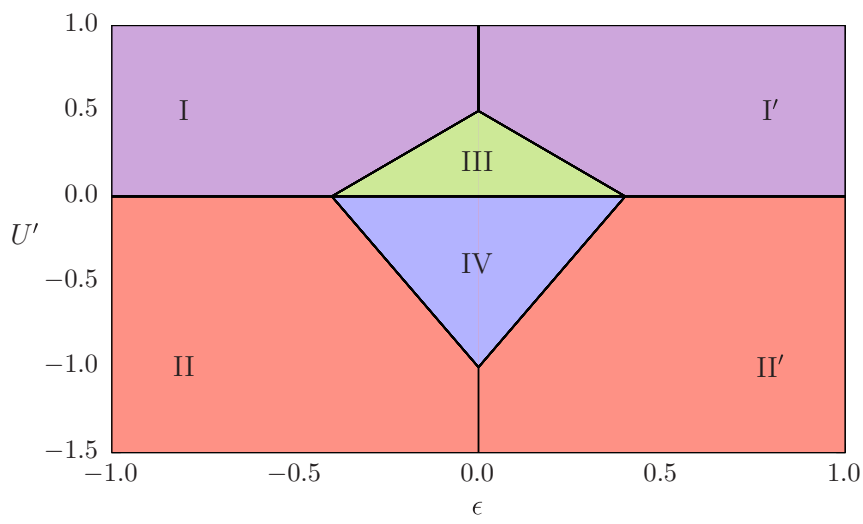

FIG. 4. (Color online) Regions of qualitatively different ground states for two particles. The outer regions correspond to double occupation of the $L$ (I,II) and $R\left(\mathrm{I}^{\prime}, \mathrm{II}^{\prime}\right)$ wells. Single occupation of both wells occur in the center region, with AF (III) and FM (IV) spin correlations.

states and to counteract the repulsion $U$ since it is attractive for the appropriate spin orientations.

By the definition (6), $C$ is zero if one of the wells is empty or doubly occupied with zero total spin, which is observed in the large $|\epsilon|$ regions of Fig. 3. In the single-occupation regime, we see that the spin correlation is negative, reflecting the fact that $U^{\prime}>0$ favors a low total spin in each well, which is consistent with tunneling to singly occupied states with opposite spins. This is similar to the mechanism of exchange interaction between localized electrons of neighboring atoms in a crystal, although in that case, due to the exclusion principle, there is no need for a spin-dependent interaction.

The results shown up to now correspond to a weak positive $U^{\prime}$. From Fig. 2 it is possible to see that the single-occupation range shrinks as $U^{\prime}$ grows. This regime disappears when $U^{\prime}$ exceeds a critical value $U_{+}^{\prime}=0.5$, since the attractive effect of $U^{\prime}$ overcomes the repulsion $U$, favoring formation of a zero-spin pair in the deeper well.

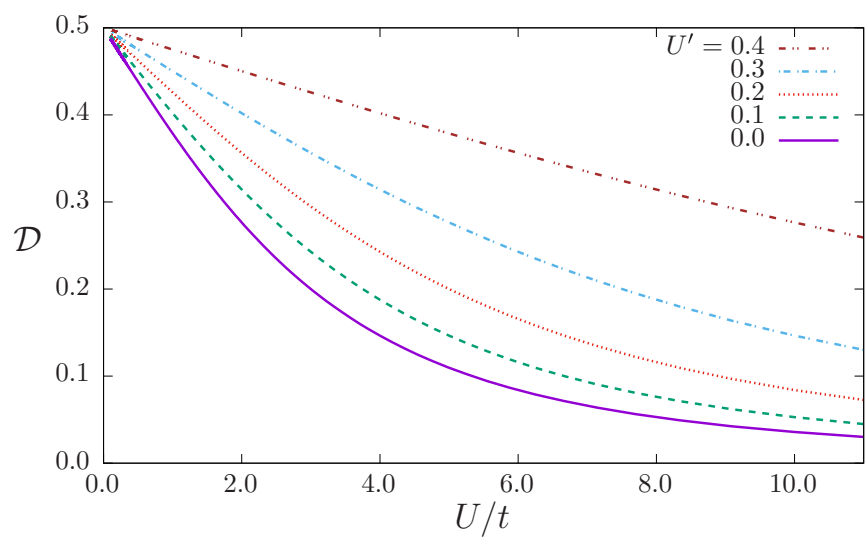

FIG. 5. (Color online) Variation of the average double occupancy as a function of the the ratio between Hubbard repulsion and tunneling strength in the two-particle ground state for a symmetric double well. The curves correspond to values of the spin-dependent interaction within the region III of Fig. 4. 


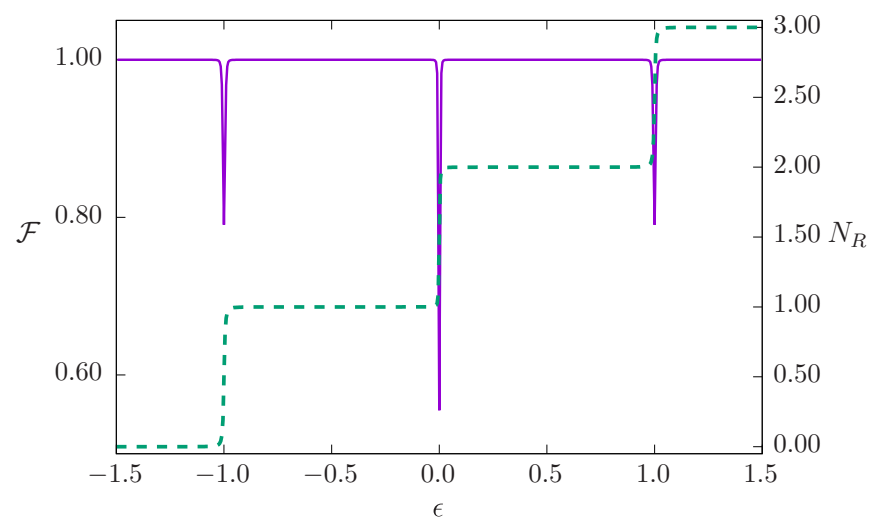

FIG. 6. (Color online) Fidelity (continuous) and $R$-well occupation (dashed) for $N_{t}=3, t=0.005$, and $U^{\prime}=0.1$.

In the case of $U^{\prime}<0$, still for a two-boson system, the spin correlation behaves similarly to what was seen for $U^{\prime}>0$ in Fig. 3, except that the sign of $C$ is reversed. Double occupancy continues to be favored by $U^{\prime}$, but now with maximum total spin in the doubly occupied well. Hence, the single-occupation region for small $\left|U^{\prime}\right|$ will show ferromagnetic correlations. The critical value for suppression of this regime is now $U_{-}^{\prime}=-1.0$ (meaning $U_{-}^{\prime}=-U$ ).

\section{Two-particle regimes in the $\left(\epsilon, U^{\prime}\right)$ plane}

It is interesting to see a complete picture of the case $N_{t}=2$, varying the asymmetry parameter $\epsilon$ and the spin-dependent interaction $U^{\prime}$, as shown in Fig. 4. The limiting lines of the various regions were obtained from fidelity minima (mostly $\mathcal{F}_{\epsilon}$, but the line at $U^{\prime}=0$ is better seen with $\mathcal{F}_{U^{\prime}}$. In regions I and II there is double occupation of the $L$ well, with $S_{L}=0$ and 2, respectively. Regions $\mathrm{I}^{\prime}$ and II' $^{\prime}$ are equivalent to I and II, but the particles are located in the $R$ side. States of one particle in each well are observed in the two central regions, III and $\mathrm{IV}$, respectively with negative and positive spin correlations. Borrowing denominations from magnetism, we can say that spin correlations are antiferromagnetic (AF) in region III and ferromagnetic (FM) in IV.

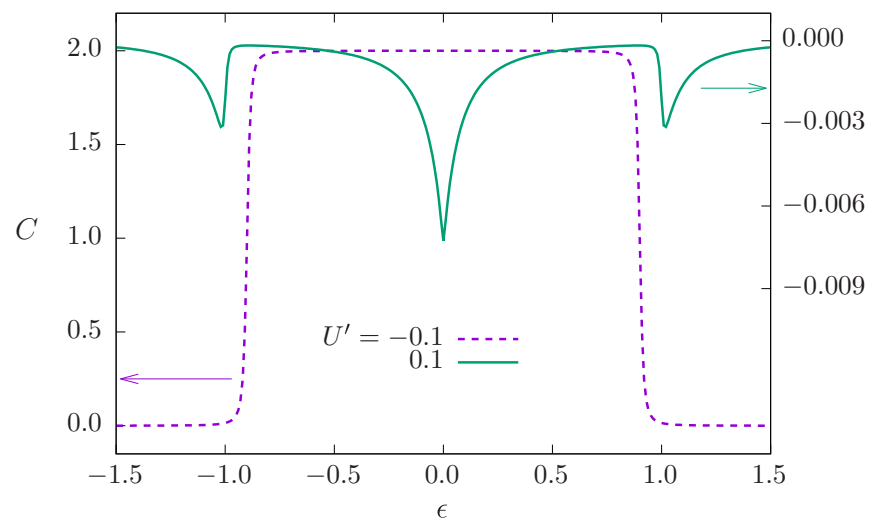

FIG. 7. (Color online) Spin correlation functions for $N_{t}=3$ and $t=0.005$ in the cases of positive and negative $U^{\prime}$. Notice the enlarged scale and displaced origin for the solid line.

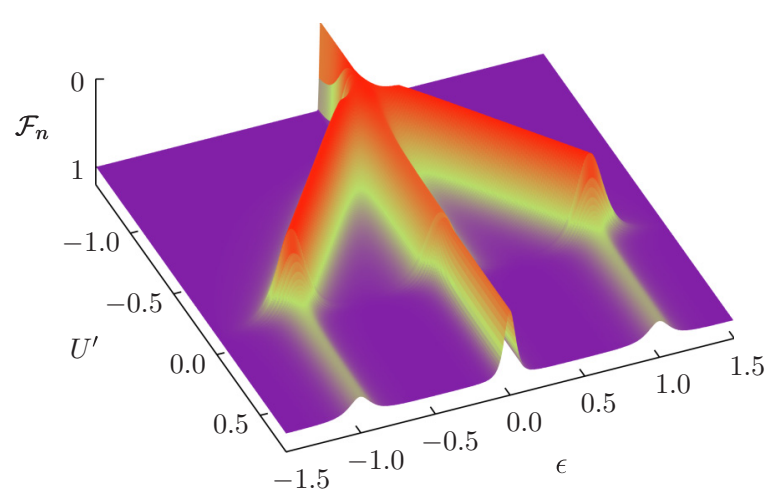

FIG. 8. (Color online) Variation of the ground-state fidelity with $\epsilon$ and $U^{\prime}$ for $t=0.1$ in a three-particle system. Notice that the fidelity axis is inverted, and the values are normalized to fall in the range $[0,1]$.

It is worth observing that the characterization of states in terms of doubly or singly occupied wells refers to the average occupation. Fluctuations occur for any $t \neq 0$, so that the sharp boundary lines of Fig. 4 are only sharp for very weak tunneling. For instance, by choosing a favorable condition for single occupancy inside region III (e.g., symmetric wells and small $\left|U^{\prime}\right|$ ), a possible measure of the probability of double occupancy is given by $\mathcal{D} \equiv 1-\mathcal{F}_{t}^{2}$, where $\mathcal{F}_{t}$ is the fidelity between the ground states with zero and nonzero tunneling. This is shown as a function of $U / t$ in Fig. 5, where we can see that it is null for decoupled wells and grows with the tunneling strength, approaching $1 / 2$ for large $t$, when all possible occupancies become energetically equivalent. The bottom curve $\left(U^{\prime}=0\right)$ is very similar to the one obtained for two fermions in a double well [29]. Here, the effect of increasing $U^{\prime}$ is to lower the energy of doubly occupied wells.

\section{THREE OR MORE PARTICLES}

Adding a third particle to the system leads to noticeable qualitative changes. Figure 6 shows the fidelity and $R$-well

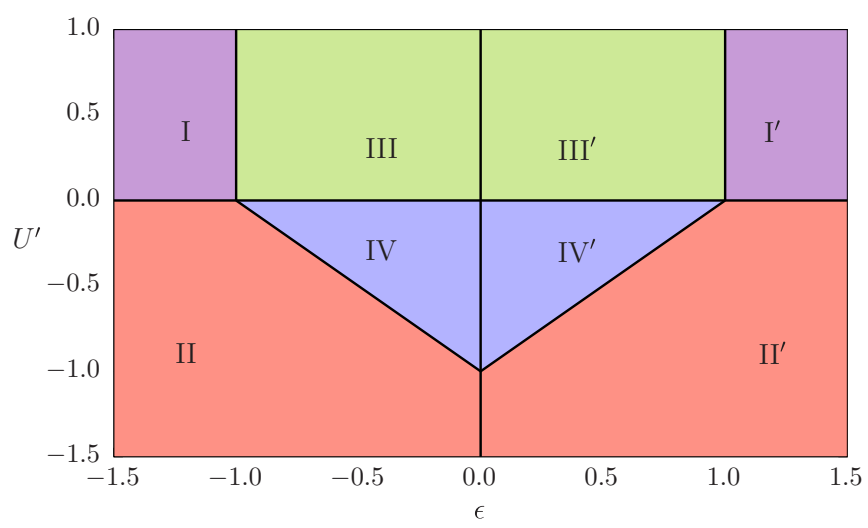

FIG. 9. (Color online) Regions of qualitatively different ground states for three particles. The outer regions correspond to double occupation of the $L(\mathrm{I}, \mathrm{II})$ and $R\left(\mathrm{I}^{\prime}, \mathrm{II}^{\prime}\right)$ wells. In the central regions (III, III', IV, IV') there is single occupation in one well and double in the other, with very weak AF correlations for $U^{\prime}>0$ and strong FM correlations for $U^{\prime}<0$. 

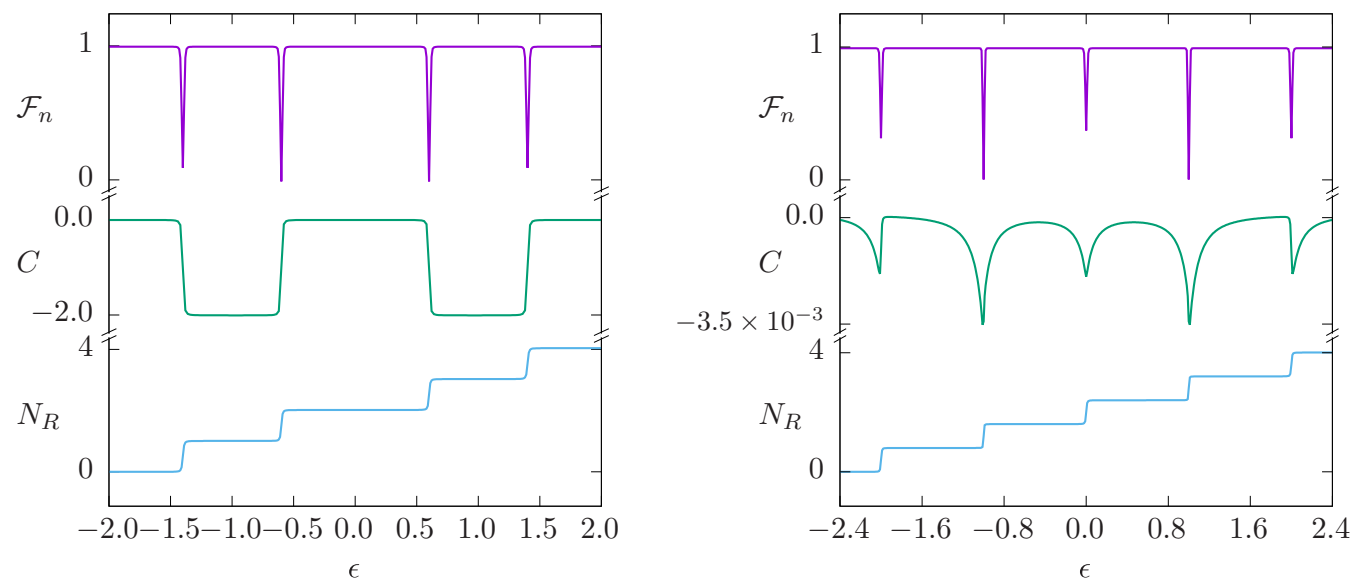

FIG. 10. (Color online) Normalized fidelity $\left(\mathcal{F}_{n}\right)$, spin correlation function $(C)$, and $R$-well occupation for systems of four particles (left panel) and five particles (right panel), with $t=0.005$ and $U^{\prime}=0.1$.

occupation for varying $\epsilon$. The plot is for $U^{\prime}>0$, but the behavior for negative $U^{\prime}$ is qualitatively the same. Notice that there is a central minimum of the fidelity, corresponding to the extra step observed for $N_{R}$, but no central plateau, in contrast with Fig. 2, since it is impossible to have the same number of particles in both wells. The most striking difference comes from the spin correlation function, shown in Fig. 7. For positive $U^{\prime}$ the correlation is negative but close to zero except, for a slight increase in magnitude near the changes of ground state. This is due to the fact that there is always either zero or two particles in one of the wells, which means that one of the spins is always essentially null. In contrast, for $U^{\prime}<0$ the whole central region, where none of the wells is empty, presents a large positive correlation between the spins of the two wells.

As in the two-particle case, when the magnitude of a negative $U^{\prime}$ grows beyond a critical value (once more, $U_{-}^{\prime}=-1$ ), all three particles stick together in one of the wells (with maximum spin $S_{i}=3$ ), so that the two inner regions around the central minimum in Fig. 6 cease to exist. On the other hand, for positive $U^{\prime}$ the fidelity minima are located at the same $\epsilon$ values seen in Fig. 6, independent of $U^{\prime}$. This is shown in Fig. 8, where we plot a normalized fidelity $\mathcal{F}_{n}$ (rescaled to fall in the range $[0,1]$ ) as a function of both $\epsilon$ and $U^{\prime}$. The observed independence on $U^{\prime}$ is due to the constraint that $N_{i}+S_{i}$ must be even, which means that the minimum total spin in a given well is $S_{i}=0$ for $N_{i}=2$ and $S_{i}=1$ for $N_{i}=3$. It then follows that the eigenvalue associated with the $U^{\prime}$ term [see Eq. (1)] is $S_{i}\left(S_{i}+1\right)-2 N_{i}=-4$ in both cases, so that the change in ground state is driven by a balance between $\epsilon$ and the local repulsion $U$.

A ground-state diagram like that of Fig. 4 can be built for $N=3$, as shown in Fig. 9. Here too the regions labeled with primed roman numbers are equivalent to the corresponding unprimed ones upon the exchange $L \leftrightarrow R$. In I and II we have essentially three particles in $L$ while $R$ is nearly empty, with $S_{t}=3$ in I and $S_{t}=1$ in II. Regions III and IV correspond to double occupation of $L$ and the third particle in $R$, with the spin states compatible with spin correlations as shown in Fig. 7.

\section{Larger $N$}

The main trends in behavior observed for two and three particles appear in general for even and odd particle numbers. This is exemplified in Fig. 10 for $N_{t}=4$ and 5. Obviously, the staircase increase in single-well average occupation has a number of steps reflecting the total particle number. Each step is accompanied by a sharp minimum of the fidelity.

Spin correlation functions are shown in Fig. 10 only for $U^{\prime}>0$. They reproduce the scenarios already viewed for two and three particles. Also similarly to those cases, correlations for $U^{\prime}<0$ are positive (when not null), and have significant values that remain nearly constant between jumps of singlewell occupations.

The "phase diagrams" are similar to Fig. 4 for even $N_{t}$ and to Fig. 9 for odd $N_{t}$, except that the central part has a growing number of nested regions as the number of possible distributed occupations increases.

\section{CONCLUSIONS}

We studied the possible ground states of spin-1 bosons in double-well potentials. Such systems can model the basic unit of optical lattices with trapped cold atoms, for which parameters such as the depth of the wells, amplitude of tunneling between them, and interactions between particles can be controlled. We based our analysis on the usual Bose-Hubbard Hamiltonian with an additional on-site spindependent interaction, as proposed in Ref. [23].

Even considering different total numbers of particles, restriction to two wells allowed us to exactly diagonalize the Hamiltonian matrix in relevant subspaces and to study changes of the ground state induced by variation of the model parameters. Our analysis focused primarily on a regime of weak tunneling relative to the local repulsive (Hubbard) interaction, which we kept fixed. The variable parameters were then the relative depth between the two wells and the local spin-dependent coupling, which we allowed to be positive or negative, respectively, favoring low and high total spin in each well. 
We showed that regime changes in the system can be detected by evaluating the ground-state fidelity as some parameter is varied. This quantity presents sharp minima at parameter values for which the nature of the ground state changes, which should correspond to critical values for occurrence of quantum phase transitions in the macroscopic limit. Information on the nature of the different ground states has to be sought through evaluation of average values of appropriate physical quantities, like the number of particles and total spin in a given well or interwell spin correlations.

The results allowed us to construct maps of different regimes in parameter space, identifying regions of full occupancy of a single well, and regions with particles distributed in both wells, in which case either ferromagnetic or antiferromagnetic spin correlations between the two wells occur as a consequence of the spin-dependent interaction. This was done in detail for system of two and three particles. Comparison with some results for four and five particles revealed that the main qualitative differences occur between even and odd total number of particles in the system.

This study can be extended to larger numbers, not only of particles but also of wells, the limitations being only computational. Work in dynamical processes in the same model is now in progress, including investigation of spin effects on transistor-like behavior [43] in the three-well case. Additionally, by increasing the number of wells we can address the problem of Anderson localization [44] with a distribution of well depths, taking into account the effect of nonzero spin and spin-dependent interactions.

\section{ACKNOWLEDGMENTS}

A.F. thanks A. Wagner for sharing important information and acknowledges invaluable discussions with S. Jochim. This work was supported in part by the Brazilian agencies Conselho Nacional de Desenvolvimento Científico e Tecnológico (CNPq) and Coordenação de Aperfeicoamento de Pessoal de Nível Superior (CAPES).
[1] M. H. Anderson, J. R. Ensher, M. R. Matthews, C. E. Wieman, and E. A. Cornell, Science 269, 198 (1995).

[2] C. C. Bradley, C. A. Sackett, J. J. Tollett, and R. G. Hulet, Phys. Rev. Lett. 75, 1687 (1995).

[3] K. B. Davis, M.-O. Mewes, M. R. Andrews, N. J. van Druten, D. S. Durfee, D. M. Kurn, and W. Ketterle, Phys. Rev. Lett. 75, 3969 (1995).

[4] D. M. Stamper-Kurn, M. R. Andrews, A. P. Chikkatur, S. Inouye, H.-J. Miesner, J. Stenger, and W. Ketterle, Phys. Rev. Lett. 80, 2027 (1998).

[5] H.-J. Miesner, D. M. Stamper-Kurn, J. Stenger, S. Inouye, A. P. Chikkatur, and W. Ketterle, Phys. Rev. Lett. 82, 2228 (1999).

[6] M. R. Matthews, B. P. Anderson, P. C. Haljan, D. S. Hall, C. E. Wieman, and E. A. Cornell, Phys. Rev. Lett. 83, 2498 (1999).

[7] M. D. Barrett, J. A. Sauer, and M. S. Chapman, Phys. Rev. Lett. 87, 010404 (2001).

[8] M.-S. Chang, Q. Qin, W. Zhang, L. You, and M. S. Chapman, Nat. Phys. 1, 111 (2005).

[9] T.-L. Ho, Phys. Rev. Lett. 81, 742 (1998).

[10] T. Ohmi and K. Machida, J. Phys. Soc. Jpn. 67, 1822 (1998).

[11] A. Imambekov, M. Lukin, and E. Demler, Phys. Rev. A 68, 063602 (2003).

[12] S. Tsuchiya, S. Kurihara, and T. Kimura, Phys. Rev. A 70, 043628 (2004).

[13] K. V. Krutitsky and R. Graham, Phys. Rev. A 70, 063610 (2004).

[14] T. Kimura, S. Tsuchiya, and S. Kurihara, Phys. Rev. Lett. 94, 110403 (2005).

[15] K. V. Krutitsky, M. Timmer, and R. Graham, Phys. Rev. A 71, 033623 (2005).

[16] Ö. E. Müstecaplığlu, W. Zhang, and L. You, Phys. Rev. A 75, 023605 (2007).

[17] R. V. Pai, K. Sheshadri, and R. Pandit, Phys. Rev. B 77, 014503 (2008).

[18] B. Juliá-Díaz, M. Melé-Messeguer, M. Guilleumas, and A. Polls, Phys. Rev. A 80, 043622 (2009).
[19] M. Melé-Messeguer, S. Paganelli, B. Juliá-Díaz, A. Sanpera, and A. Polls, Phys. Rev. A 86, 053626 (2012).

[20] M. Melé-Messeguer, B. Juliá-Díaz, A. Polls, and L. Santos, Phys. Rev. A 87, 033632 (2013).

[21] C. C. N. Kuhn, X. W. Guan, A. Foerster, and M. T. Batchelor, Phys. Rev. A 85, 043606 (2012).

[22] C. C. N. Kuhn, X. W. Guan, A. Foerster, and M. T. Batchelor, Phys. Rev. A 86, 011605(R) (2012).

[23] A. Wagner, C. Bruder, and E. Demler, Phys. Rev. A 84, 063636 (2011).

[24] A. Wagner, A. Nunnenkamp, and C. Bruder, Phys. Rev. A 86, 023624 (2012).

[25] D. Jaksch, C. Bruder, J. I. Cirac, C. W. Gardiner, and P. Zoller, Phys. Rev. Lett. 81, 3108 (1998).

[26] F. Serwane, G. Zürn, T. Lompe, T. B. Ottenstein, A. N. Wenz, and S. Jochim, Science 332, 6027 (2011).

[27] G. Zürn, F. Serwane, T. Lompe, A. N. Wenz, M. G. Ries, J. E. Bohn, and S. Jochim, Phys. Rev. Lett. 108, 075303 (2012).

[28] A. N. Wenz, G. Zürn, S. Murmann, I. Brouzos, T. Lompe, and S. Jochim, Science 342, 6157 (2013).

[29] S. Murmann, A. Bergschneider, V. M. Kilnkhamer, G. Zürn, T. Lompe, and S. Jochim, Phys. Rev. Lett. 114, 080402 (2015).

[30] S. Jochim (private communication).

[31] D. Blume, Rep. Prog. Phys. 75, 046401 (2012).

[32] E. Braaten and H.-W. Hammer, Phys. Rep. 428, 259 (2006).

[33] S. Zöllner, H.-D. Meyer, and P. Schmelcher, Phys. Rev. A 78, 013621 (2008).

[34] B. Chatterjee, I. Brouzos, S. Zöllner, and P. Schmelcher, Phys. Rev. A 82, 043619 (2010).

[35] I. Brouzos and P. Schmelcher, Phys. Rev. Lett. 108, 045301 (2012).

[36] N. L. Harshman, Phys. Rev. A 86, 052122 (2012).

[37] N. L. Harshman, Phys. Rev. A 89, 033633 (2014).

[38] B. Wilson, A. Foerster, C. C. N. Kuhn, I. Roditi, and D. Rubeni, Phys. Lett. A 378, 1065 (2014).

[39] I. Brouzos and A. Foerster, Phys. Rev. A 89, 053623 (2014). 
[40] N. T. Zinner, A. G. Volosniev, D. V. Fedorov, A. S. Jensen, and M. Valiente, Europhys. Lett. 107, 60003 (2014).

[41] P. D’Amico and M. Rontani, J. Phys. B: At. Mol. Opt. Phys. 47, 065303 (2014).

[42] M. A. Nielsen and I. L. Chuang, Quantum Computation and Quantum Information, Cambridge Series on Information and the Natural Sciences (Cambridge University Press, Cambridge, 2004).

[43] J. A. Stickney, D. Z. Anderson, and A. A. Zozulya, Phys. Rev. A 75, 013608 (2007).

[44] J. C. C. Cestari, A. Foerster, and M. A. Gusmão, Phys. Rev. A 82, 063634 (2010). 